International Journal of Pure and Applied Mathematics

Volume 95 No. 1 2014, 89-98

ISSN: 1311-8080 (printed version); ISSN: 1314-3395 (on-line version)

url: http://www.ijpam.eu

doi: http://dx.doi.org/10.12732/ijpam.v95i1.10

ijpam.eu

\title{
A GENERALIZATION OF DIRICHLET TYPE 1 DISTRIBUTION
}

\author{
Daya K. Nagar ${ }^{1 \S}$, Armando Gómez ${ }^{2}$ \\ ${ }^{1,2}$ Instituto de Matemáticas \\ Universidad de Antioquia \\ Calle 67, No. 53-108, Medellín, COLOMBIA
}

\begin{abstract}
In this article, we give a generalization of the Dirichlet type 1 distribution. This generalization is based on the Lauricella's type B hypergeometric function. We also study several of its properties.
\end{abstract}

AMS Subject Classification: $62 \mathrm{H} 99$

Key Words: Appell hypergeometric function, Dirichlet distribution, hypergeometric function, Lauricella function

\section{Introduction}

The random variables $U_{1}, \ldots, U_{n}$ are said to have a Dirichlet type 1 distribution with parameters $a_{1}, \ldots, a_{n} ; a_{n+1}$, denoted by $\left(U_{1}, \ldots, U_{n}\right) \sim \operatorname{D} 1\left(a_{1}, \ldots, a_{n}\right.$; $\left.a_{n+1}\right)$, if their joint probability density function (p.d.f.) is given by

$$
\begin{array}{r}
\frac{\Gamma\left(\sum_{i=1}^{n+1} a_{i}\right)}{\prod_{i=1}^{n+1} \Gamma\left(a_{i}\right)} \prod_{i=1}^{n} u_{i}^{a_{i}-1}\left(1-\sum_{i=1}^{n} u_{i}\right)^{a_{n+1}-1}, \\
u_{i}>0, \quad i=1, \ldots, n, \quad \sum_{i=1}^{n} u_{i}<1 .
\end{array}
$$

The Dirichlet type 1 distribution is primarily used as a conjugate prior distribution for the multinomial distribution parameters. Dirichlet random variables are good candidates for random weights since their beautiful stochastic representation in terms of gamma random variables and their sum is automatically

Received: April 14, 2014

(C) 2014 Academic Publications, Ltd.

$\S_{\text {Correspondence author }}$ url: www.acadpubl.eu 
one, which has applications in Bayes bootstrap method. The Dirichlet type 1 distribution has been studied extensively, for example, see Kotz, Balakrishnan and Johnson [1], and Gupta and Nagar [2].

In this article, we give a generalization of the Dirichlet type 1 distribution. This generalization is based on the Lauricella type B hypergeometric function and thus will be called Dirichlet-Lauricella type B distribution. In Section 2, we give definitions of Lauricella type B and type D hypergeometric functions. We define Dirichlet-Lauricella type B distribution in Section 3. Section 4, deals with several properties such as marginal densities and joint moment.

\section{Preliminaries}

The Pochhammer symbol $(a)_{n}$ is defined by $(a)_{n}=a(a+1) \cdots(a+n-1)$ $=(a)_{n-1}(a+n-1)$ for $n=1,2, \ldots$ and $(a)_{0}=1$. The Appell-Lauricella hypergeometric functions of several variable are generalizations of the classical hypergeometric function of one variable. Appell's name is usually associated to the two variable case, whereas the case of more variables is usually ascribed to Lauricella. In this section, we define the Lauricella hypergeometric functions $F_{B}^{(n)}$ and $F_{D}^{(n)}$ of several variables. For further results and properties of these functions the reader is referred to Srivastava and Karlsson [8], and Prudnikov, Brychkov and Marichev [7, Sec. 7.2.4]. The Lauricella hypergeometric functions $F_{B}^{(n)}$ and $F_{D}^{(n)}$ are defined in multiple series as

$$
\begin{aligned}
& F_{B}^{(n)}\left(a_{1}, \ldots, a_{n}, b_{1}, \ldots, b_{n} ; c ; z_{1}, \ldots, z_{n}\right) \\
& =\sum_{j_{1}, \ldots, j_{n}=0}^{\infty} \frac{\left(a_{1}\right)_{j_{1}} \cdots\left(a_{n}\right)_{j_{n}}\left(b_{1}\right)_{j_{1}} \cdots\left(b_{n}\right)_{j_{n}}}{(c)_{j_{1}+\cdots+j_{n}}} \frac{z_{1}^{j_{1}} \cdots z_{n}^{j_{n}}}{j_{1} ! \cdots j_{n} !}, \max \left\{\left|z_{1}\right|, \ldots,\left|z_{n}\right|\right\}<1
\end{aligned}
$$

and

$$
\begin{aligned}
& F_{D}^{(n)}\left(a, b_{1}, \ldots, b_{n} ; c ; z_{1}, \ldots, z_{n}\right) \\
& =\sum_{j_{1}, \ldots, j_{n}=0}^{\infty} \frac{(a)_{j_{1}+\cdots+j_{n}}\left(b_{1}\right)_{j_{1}} \cdots\left(b_{n}\right)_{j_{n}}}{(c)_{j_{1}+\cdots+j_{n}}} \frac{z_{1}^{j_{1}} \cdots z_{n}^{j_{n}}}{j_{1} ! \cdots j_{n} !}, \quad \max \left\{\left|z_{1}\right|, \ldots,\left|z_{n}\right|\right\}<1,
\end{aligned}
$$

respectively.

If $n=2$, then these functions reduce to Appell hypergeometric functions $F_{3}$ and $F_{1}$, respectively. For $n=1$, they reduce to the Gauss hypergeometric function ${ }_{2} F_{1}$. 
By writing $(c)_{j_{1}+\cdots+j_{n}}=(c)_{j_{r+1}+\cdots+j_{n}}\left(c+j_{r+1}+\cdots+j_{n}\right)_{j_{1}+\cdots+j_{r}}, 1 \leq r \leq n$, in (2.1), the Appell hypergeometric function $F_{B}^{(n)}$ can also be expressed as

$$
\begin{aligned}
& F_{B}^{(n)}\left(a_{1}, \ldots, a_{n}, b_{1}, \ldots, b_{n} ; c ; z_{1}, \ldots, z_{n}\right) \\
& =\sum^{\infty} \frac{\left(a_{r+1}\right)_{j_{r+1}} \cdots\left(a_{n}\right)_{j_{n}}\left(b_{r+1}\right)_{j_{r+1}} \cdots\left(b_{n}\right)_{j_{n}}}{(c)_{j_{r+1}+\cdots+j_{n}}} \frac{z_{r+1}^{j_{r+1}} \cdots, j_{n}=0}{j_{r+1} ! \cdots j_{n} !} \\
& \quad \times F_{B}^{(r)}\left(a_{1}, \ldots, a_{r}, b_{1}, \ldots, b_{r} ; c+j_{r+1}+\cdots+j_{n} ; z_{1}, \ldots, z_{r}\right) .
\end{aligned}
$$

The integral representation of $F_{B}^{(n)}$ is given by

$$
\begin{aligned}
& F_{B}^{(n)}\left(a_{1}, \ldots, a_{n}, b_{1}, \ldots, b_{n} ; c ; z_{1}, \ldots, z_{n}\right)= \\
& \frac{\Gamma(c)}{\prod_{i=1}^{n} \Gamma\left(a_{i}\right) \Gamma\left(c-\sum_{i=1}^{n} a_{i}\right)} \int_{\substack{u_{1}>0, \ldots, u_{n}>0 \\
1-\sum_{i=1}^{n} u_{i}>0}} \ldots \int \frac{\prod_{i=1}^{n} u_{i}^{a_{i}-1}\left(1-\sum_{i=1}^{n} u_{i}\right)^{c-\sum_{i=1}^{n} a_{i}-1}}{\prod_{i=1}^{n}\left(1-z_{i} u_{i}\right)^{b_{i}}} \prod_{i=1}^{n} \mathrm{~d} u_{i},
\end{aligned}
$$

where $\operatorname{Re}\left(a_{i}\right)>0, i=1, \ldots, n, \operatorname{Re}\left(c-a_{1}-\cdots-a_{n}\right)>0$ and $\left|\arg \left(1-z_{i}\right)\right|<\pi$, $i=1, \ldots, n$.

The Lauricalla hypergeometric function $F_{D}$ has integral representation

$$
F_{D}^{(n)}\left(a, b_{1}, \ldots, b_{n} ; c ; z_{1}, \ldots, z_{n}\right)=\frac{\Gamma(c)}{\Gamma(a) \Gamma(c-a)} \int_{0}^{1} \frac{u^{a-1}(1-u)^{c-a-1}}{\prod_{i=1}^{n}\left(1-u z_{i}\right)^{b_{i}}} \mathrm{~d} u
$$

where $\operatorname{Re}(c)>\operatorname{Re}(a)>0$ and $\left|\arg \left(1-z_{i}\right)\right|<\pi, i=1, \ldots, n$.

\section{The Dirichlet-Lauricella Type B Distribution}

We define the Dirichlet-Lauricella type B distribution as follows.

The random variables $U_{1}, \ldots, U_{n}$ are said to have a Dirichlet-Lauricella type $\mathrm{B}$ distribution with parameters $a_{1}, \ldots, a_{n} ; c ; d_{1}, \ldots, d_{n} ; \theta_{1}, \ldots, \theta_{n}$, denoted by $\left(U_{1}, \ldots, U_{n}\right) \sim \operatorname{DLB}\left(a_{1}, \ldots, a_{n} ; c ; d_{1}, \ldots, d_{n} ; \theta_{1}, \ldots, \theta_{n}\right)$, if their joint p.d.f. is given by

$$
K_{B} \frac{\prod_{i=1}^{n} u_{i}^{a_{i}-1}\left(1-\sum_{i=1}^{n} u_{i}\right)^{c-\sum_{i=1}^{n} a_{i}-1}}{\prod_{i=1}^{n}\left(1-\theta_{i} u_{i}\right)^{d_{i}}}, u_{i}>0, i=1, \cdots, n, \sum_{i=1}^{n} u_{i}<1,
$$


where $a_{1}>0, \ldots, a_{n}>0, c-\sum_{i=1}^{n} a_{i}>0$ and $\left|\theta_{i}\right|<1, i=1, \ldots, n$. The normalizing constant $K_{B}$ in (3.1) is given by

$$
\begin{aligned}
K_{B}^{-1} & =\int_{\substack{u_{1}>0, \ldots, u_{n}>0 \\
\sum_{i=1}^{n} u_{i}<1}} \frac{\prod_{i=1}^{n} u_{i}^{a_{i}-1}\left(1-\sum_{i=1}^{n} u_{i}\right)^{c-\sum_{i=1}^{n} a_{i}-1}}{\prod_{i=1}^{n}\left(1-\theta_{i} u_{i}\right)^{d_{i}}} \prod_{i=1}^{n} \mathrm{~d} u_{i} \\
& =\frac{\prod_{i=1}^{n} \Gamma\left(a_{i}\right) \Gamma\left(c-\sum_{i=1}^{n} a_{i}\right)}{\Gamma(c)} F_{B}^{(n)}\left(a_{1}, \ldots, a_{n}, d_{1}, \ldots, d_{n} ; c ; \theta_{1}, \ldots, \theta_{n}\right),
\end{aligned}
$$

where the last line has been obtained by using (2.4). For $n=1$, the above p.d.f. reduces to a generalized beta type 1 p.d.f. given by

$$
\frac{\Gamma(c)}{\Gamma\left(a_{1}\right) \Gamma\left(c-a_{1}\right){ }_{2} F_{1}\left(a_{1}, d_{1} ; c ; \theta_{1}\right)} \frac{u_{1}^{a_{1}-1}\left(1-u_{1}\right)^{c-a_{1}-1}}{\left(1-\theta_{1} u_{1}\right)^{d_{1}}}, 0<u_{1}<1,
$$

where $c>a_{1}>0,-1<\theta_{1}<1$ and ${ }_{2} F_{1}$ is the Gauss hypergeometric function. The generalized beta type 1 distribution has been studied by Nagar and RadaMora [5], Libby and Novic [3], Pham-Gia and Duong [6]. Further, for $n=2$, the p.d.f. in (3.1) slides to a generalized bivariate beta type 1 p.d.f. defined by (Nadarajah [4]),

$$
\begin{aligned}
& \frac{\Gamma(c)}{\Gamma\left(a_{1}\right) \Gamma\left(a_{2}\right) \Gamma\left(c-a_{1}-a_{2}\right) F_{3}\left(a_{1}, a_{2}, d_{1}, d_{2} ; c ; \theta_{1}, \theta_{2}\right)} \\
& \times \frac{u_{1}^{a_{1}-1} u_{2}^{a_{2}-1}\left(1-u_{1}-u_{2}\right)^{c-a_{1}-a_{2}-1}}{\left(1-\theta_{1} u_{1}\right)^{d_{1}}\left(1-\theta_{2} u_{2}\right)^{d_{2}}}, u_{1}>0, u_{2}>0, u_{1}+u_{2}<1,
\end{aligned}
$$

where $a_{1}>0, a_{2}>0, c>a_{1}+a_{2},-1<\theta_{1}<1,-1<\theta_{2}<1$ and $F_{3}$ is the third hypergeometric function of Appell.

\section{Properties}

This section gives several properties of the Dirichlet-Lauricella type B distribution.

Consider the transformation $Z_{i}=\left(1-\sum_{j=1}^{s} U_{j}\right)^{-1} U_{i}, i=s+1, \ldots, n$. Then, $u_{i}=\left(1-\sum_{j=1}^{s} u_{j}\right) z_{i}, i=s+1, \ldots, n$ with the Jacobian $J\left(u_{s+1}, \ldots, u_{n} \rightarrow\right.$ $\left.z_{s+1}, \ldots, z_{n}\right)=\left(1-\sum_{j=1}^{s} u_{j}\right)^{n-s}$. Substituting appropriately in (3.1), the joint p.d.f. of $U_{1}, \ldots, U_{s}, Z_{s+1}, \ldots, Z_{n}$ is given by

$K_{B} \frac{\prod_{i=1}^{s} u_{i}^{a_{i}-1}\left(1-\sum_{i=1}^{s} u_{i}\right)^{c-\sum_{i=1}^{s} a_{i}-1}}{\prod_{i=1}^{s}\left(1-\theta_{i} u_{i}\right)^{d_{i}}} \frac{\prod_{i=s+1}^{n} z_{i}^{a_{i}-1}\left(1-\sum_{i=s+1}^{n} z_{i}\right)^{c-\sum_{i=1}^{n} a_{i}-1}}{\prod_{i=s+1}^{n}\left[1-\theta_{i} z_{i}\left(1-\sum_{j=1}^{s} u_{j}\right)\right]^{d_{i}}}$, 
where $u_{i}>0, i=1, \ldots, s, \sum_{i=1}^{s} u_{i}<1, z_{i}>0, i=s+1, \ldots, n$ and $\sum_{i=s+1}^{n} z_{i}<$ 1.

First, we find the marginal p.d.f. of $U_{1}, \ldots, U_{s}$ by integrating out $z_{s+1}, \ldots, z_{n}$ from the joint p.d.f. of $U_{1}, \ldots, U_{s}, Z_{s+1}, \ldots, Z_{n}$ as

$$
\begin{aligned}
& K_{B} \frac{\prod_{i=1}^{s} u_{i}^{a_{i}-1}\left(1-\sum_{i=1}^{s} u_{i}\right)^{c-\sum_{i=1}^{s} a_{i}-1}}{\prod_{i=1}^{s}\left(1-\theta_{i} u_{i}\right)^{d_{i}}} \\
& \times \int_{\substack{z_{s+1}>0, \ldots, z_{n}>0 \\
\sum_{i=s+1}^{n} z_{i}<1}} \frac{\prod_{i=s+1}^{n} z_{i}^{a_{i}-1}\left(1-\sum_{i=s+1}^{n} z_{i}\right)^{c-\sum_{i=1}^{n} a_{i}-1}}{\prod_{i=s+1}^{n}\left[1-\theta_{i} z_{i}\left(1-\sum_{j=1}^{s} u_{j}\right)\right]^{d_{i}}} \prod_{i=s+1}^{n} \mathrm{~d} z_{i} .
\end{aligned}
$$

Now, using the integral representation of $F_{B}^{(n)}$ given in (2.4), we derive the marginal p.d.f. of $U_{1}, \ldots, U_{s}$ as

$$
\begin{gathered}
K_{B 1} \frac{\prod_{i=1}^{s} u_{i}^{a_{i}-1}\left(1-\sum_{i=1}^{s} u_{i}\right)^{c-\sum_{i=1}^{s} a_{i}-1}}{\prod_{i=1}^{s}\left(1-\theta_{i} u_{i}\right)^{d_{i}}} F_{B}^{(n-s)}\left(a_{s+1}, \ldots, a_{n}, d_{s+1}, \ldots, d_{n}\right. \\
\left.c-\sum_{i=1}^{s} a_{i} ; \theta_{s+1}\left(1-\sum_{i=1}^{s} u_{i}\right), \ldots, \theta_{n}\left(1-\sum_{i=1}^{s} u_{i}\right)\right)
\end{gathered}
$$

where $u_{i}>0, i=1, \ldots, s, \sum_{i=1}^{s} u_{i}<1$ and

$$
K_{B 1}^{-1}=\frac{\prod_{i=1}^{s} \Gamma\left(a_{i}\right) \Gamma\left(c-\sum_{i=1}^{s} a_{i}\right)}{\Gamma(c)} F_{B}^{(n)}\left(a_{1}, \ldots, a_{n}, d_{1}, \ldots, d_{n} ; c ; \theta_{1}, \ldots, \theta_{n}\right) .
$$

It is interesting to note that the marginal p.d.f. of $U_{1}, \ldots, U_{s}$ does not belong to the Dirichlet-Lauricella type B family of distributions and differs by an additional factor containing the Lauricella hypergeometric function $F_{B}$.

Further, making the transformation $Z_{i}=\left(1-U_{s}\right)^{-1} U_{i}, i=1, \ldots, s-1$ with the Jacobian $J\left(u_{1}, \ldots, u_{s-1} \rightarrow z_{1}, \ldots, z_{s-1}\right)=\left(1-u_{s}\right)^{s-1}$ in (4.3), the joint p.d.f. of $Z_{1}, \ldots, Z_{s-1}$ and $U_{s}$ is derived as

$$
\begin{aligned}
& K_{B 1} \frac{u_{s}^{a_{s}-1}\left(1-u_{s}\right)^{c-a_{s}-1}}{\left(1-\theta_{s} u_{s}\right)} \frac{\prod_{i=1}^{s-1} z_{i}^{a_{i}-1}\left(1-\sum_{i=1}^{s-1} z_{i}\right)^{c-\sum_{i=1}^{s} a_{i}-1}}{\prod_{i=1}^{s-1}\left[1-\theta_{i} z_{i}\left(1-u_{s}\right)\right]^{d_{i}}} \\
& \times F_{B}^{(n-s)}\left(a_{s+1}, \ldots, a_{n}, d_{s+1}, \ldots, d_{n} ; c-\sum_{i=1}^{s} a_{i} ;\right. \\
& \left.\quad \theta_{s+1}\left(1-u_{s}\right)\left(1-\sum_{i=1}^{s-1} z_{i}\right), \ldots, \theta_{n}\left(1-u_{s}\right)\left(1-\sum_{i=1}^{s-1} z_{i}\right)\right) .
\end{aligned}
$$


where $0<u_{s}<1, z_{i}>0, i=1, \ldots, s-1$, and $\sum_{i=1}^{s-1} z_{i}<1$. Now, expanding $F_{B}^{(n-s)}$ using (2.1) and integrating $u_{1}, \ldots, u_{s-1}$ by applying (2.4) in the above p.d.f., the marginal p.d.f. of $U_{s}$ is derived as

$$
\begin{aligned}
& K_{B 2} \frac{u_{s}^{a_{s}-1}\left(1-u_{s}\right)^{c-a_{s}-1}}{\left(1-\theta_{s} u_{s}\right)} \sum_{j_{s+1}, \ldots, j_{n}=0}^{\infty} \frac{\left(a_{s+1}\right)_{j_{s+1}} \cdots\left(a_{n}\right)_{j_{n}}\left(d_{s+1}\right)_{j_{s+1}} \cdots\left(d_{n}\right)_{j_{n}}}{\left(c-a_{s}\right)_{j_{s+1}}+\cdots+j_{n}} \\
& \times \frac{\theta_{s+1}^{j_{s+1}} \cdots \theta_{n}^{j_{n}}}{j_{s+1} ! \cdots j_{n} !}\left(1-u_{s}\right)^{j_{s+1}+\cdots+j_{n}} F_{B}^{(s-1)}\left(a_{1}, \ldots, a_{s-1}, d_{1}, \ldots, d_{s-1} ;\right. \\
& \left.c+j_{s+1}+\cdots+j_{n}-a_{s} ; \theta_{1}\left(1-u_{s}\right), \ldots, \theta_{n}\left(1-u_{s}\right)\right),
\end{aligned}
$$

where

$$
K_{B 2}^{-1}=\frac{\Gamma\left(a_{s}\right) \Gamma\left(c-a_{s}\right)}{\Gamma(c)} F_{B}^{(n)}\left(a_{1}, \ldots, a_{n}, d_{1}, \ldots, d_{n} ; c ; \theta_{1}, \ldots, \theta_{n}\right) .
$$

Finally, rewriting the infinite series involving the Lauricella function by using (2.3), the p.d.f. of $U_{s}$ is derived as

$$
\begin{gathered}
K_{B 2} \frac{u_{s}^{a_{s}-1}\left(1-u_{s}\right)^{c-a_{s}-1}}{\left(1-\theta_{s} u_{s}\right)^{d_{s}}} F_{B}^{(n-1)}\left(a_{1}, \ldots, a_{s-1}, a_{s+1}, \ldots, a_{n}, d_{1}, \ldots, d_{s-1}, d_{s+1}, \ldots, d_{n}\right. \\
\left.c-a_{s} ; \theta_{1}\left(1-u_{s}\right), \ldots, \theta_{s-1}\left(1-u_{s}\right), \theta_{s+1}\left(1-u_{s}\right), \ldots, \theta_{n}\left(1-u_{s}\right)\right) .
\end{gathered}
$$

The marginal joint p.d.f. of $Z_{s+1}, \ldots, Z_{n}$ is given by

$$
\begin{aligned}
& K_{B} \prod_{i=s+1}^{n} z_{i}^{a_{i}-1}\left(1-\sum_{i=s+1}^{n} z_{i}\right)^{c-\sum_{i=1}^{n} a_{i}-1} \\
& \quad \times \int_{\substack{u_{1}>0, \ldots, u_{s}>0 \\
\sum_{i=1}^{s} u_{i}<1}} \ldots \int_{i=1}^{s} \frac{\prod_{i=1}^{s} u_{i}^{a_{i}-1}\left(1-\sum_{i=1}^{s} u_{i}\right)^{c-\sum_{i=1}^{s} a_{i}-1}}{\left.\prod_{i}^{s} u_{i}\right)^{d_{i}} \prod_{i=s+1}^{n}\left[1-\theta_{i} z_{i}\left(1-\sum_{i=1}^{s} u_{i}\right)\right]^{d_{i}}} \prod_{i=1}^{s} \mathrm{~d} u_{i} .
\end{aligned}
$$

Now, writing

$$
\begin{aligned}
& \prod_{i=s+1}^{n}\left[1-\theta_{i} z_{i}\left(1-\sum_{i=1}^{s} u_{i}\right)\right]^{-d_{i}} \\
& =\sum_{j_{s+1}, \ldots, j_{n}=0}^{\infty} \frac{\left(d_{s+1}\right)_{j_{s+1}} \cdots\left(d_{n}\right)_{j_{n}}\left(\theta_{s+1} z_{s+1}\right)^{j_{s+1} \cdots\left(\theta_{n} z_{n}\right)^{j_{n}}}}{j_{s+1} ! \cdots j_{n} !}\left(1-\sum_{i=1}^{s} u_{i}\right)^{j_{s+1}+\cdots+j_{n}}
\end{aligned}
$$


and integrating $u_{1}, \ldots, u_{s-1}$ by applying (2.4) in the above p.d.f., the marginal p.d.f. of $Z_{s+1}, \ldots, Z_{n}$ is derived as

$$
\begin{aligned}
& K_{B 3} \prod_{i=s+1}^{n} z_{i}^{a_{i}-1}\left(1-\sum_{i=s+1}^{n} z_{i}\right)^{c-\sum_{i=1}^{n} a_{i}-1} \\
& \times \sum_{\substack{j_{s+1}, \ldots, j_{n}=0 \\
\times}}^{\infty} \frac{\left(c-\sum_{i=1}^{s} a_{i}\right)_{j_{s+1}+\cdots+j_{n}}\left(d_{s+1}\right)_{j_{s+1}} \cdots\left(d_{n}\right)_{j_{n}}}{(c)_{j_{s+1}+\cdots+j_{n}}} \frac{\left(\theta_{s+1} z_{s+1}\right)^{j_{s+1}} \cdots\left(\theta_{n} z_{n}\right)^{j_{n}}}{j_{s+1} ! \cdots j_{n} !} \\
& \times F_{B}^{(s)}\left(a_{1}, \ldots, a_{s}, d_{1}, \ldots, d_{s} ; c+j_{s+1}+\cdots+j_{n} ; \theta_{1}, \ldots, \theta_{s}\right),
\end{aligned}
$$

where

$$
K_{B 3}^{-1}=\frac{\prod_{i=s+1}^{n} \Gamma\left(a_{i}\right) \Gamma\left(c-\sum_{i=1}^{n} a_{i}\right)}{\Gamma\left(c-\sum_{i=1}^{s} a_{i}\right)} F_{B}^{(n)}\left(a_{1}, \ldots, a_{n}, d_{1}, \ldots, d_{n} ; c ; \theta_{1}, \ldots, \theta_{n}\right) .
$$

It is well known that if $\left(U_{1}, \ldots, U_{n}\right) \sim \mathrm{D} 1\left(a_{1}, \ldots, a_{n} ; c-\sum_{i=1}^{n} a_{i}\right)$, then

$$
\left(\frac{U_{1}}{\sum_{i=1}^{n} U_{i}}, \ldots, \frac{U_{n-1}}{\sum_{i=1}^{n} U_{i}}\right) \sim \mathrm{D} 1\left(a_{1}, \ldots ; a_{n}\right)
$$

and the sum $\sum_{i=1}^{n} U_{i}$ follows a beta type 1 distribution with parameters $\sum_{i=1}^{n} a_{i}$ and $c-\sum_{i=1}^{n} a_{i}$. In the next theorem, we derive similar result for the DirichletLauricells type B distribution.

Theorem 4.1. Let $\left(U_{1}, \ldots, U_{n}\right) \sim \operatorname{DLB}\left(a_{1}, \ldots, a_{n} ; c ; d_{1}, \ldots, d_{n} ; \theta_{1}, \ldots, \theta_{n}\right)$ and define $U=\sum_{i=1}^{n} U_{i}$ and $X_{i}=U_{i} / U, i=1, \ldots, n-1$. Then, the joint p.d.f. of $X_{1}, \ldots, X_{n-1}$ is given as

$$
\begin{aligned}
& K_{B} \frac{\Gamma\left(\sum_{i=1}^{n} a_{i}\right) \Gamma\left(c-\sum_{i=1}^{n} a_{i}\right)}{\Gamma(c)} \prod_{i=1}^{n-1} x_{i}^{a_{i}-1}\left(1-\sum_{i=1}^{n-1} x_{i}\right)^{a_{n}-1} \\
& \times F_{D}^{(n)}\left(\sum_{i=1}^{n} a_{i}, d_{1}, \ldots, d_{n} ; c ; \theta_{1} x_{1}, \ldots, \theta_{n-1} x_{n-1}, \theta_{n}\left(1-\sum_{i=1}^{n-1} x_{i}\right)\right),
\end{aligned}
$$

where $x_{i}>0, i=1, \ldots, n-1, \sum_{i=1}^{n-1} x_{i}<1$. Further, the p.d.f. of $U$ is derived as

$$
\begin{aligned}
& K_{B} \frac{\prod_{i=1}^{n} \Gamma\left(a_{i}\right)}{\Gamma\left(\sum_{i=1}^{n} a_{i}\right)} u^{\sum_{i=1}^{n} a_{i}-1}(1-u)^{c-\sum_{i=1}^{n} a_{i}-1} \\
& \times F_{B}^{(n)}\left(a_{1}, \ldots, a_{n}, d_{1}, \ldots, d_{n} ; \sum_{i=1}^{n} a_{i} ; \theta_{1} u, \ldots, \theta_{n} u\right), \quad 0<u<1 .
\end{aligned}
$$


Proof. Substituting $u_{i}=u x_{i}, i=1, \ldots, n-1$ and $u_{n}=u\left(1-\sum_{i=1}^{n-1} x_{i}\right)$ with the Jacobian $J\left(u_{1}, \ldots, u_{n} \rightarrow x_{1}, \ldots, x_{n-1}, u\right)=u^{n-1}$ in the joint p.d.f. of $\left(U_{1}, \ldots, U_{n}\right)$, we get the joint p.d.f. of $\left(X_{1}, \ldots, X_{n-1}\right)$ and $U$ as

$$
K_{B} \prod_{i=1}^{n-1} x_{i}^{a_{i}-1}\left(1-\sum_{i=1}^{n-1} x_{i}\right)^{a_{n}-1} \frac{u^{\sum_{i=1}^{n} a_{i}-1}(1-u)^{c-\sum_{i=1}^{n} a_{i}-1}}{\prod_{i=1}^{n-1}\left(1-\theta_{i} x_{i} u\right)^{d_{i}}\left[1-\theta_{n} u\left(1-\sum_{i=1}^{n-1} x_{i}\right)\right]^{d_{n}}},
$$

where $x_{i}>0, i=1, \ldots, n-1, \sum_{i=1}^{n-1} x_{i}<1$ and $0<u<1$. Now, integrating $u$ in the above expression using the integral representation of $F_{D}$ given in (2.5), we get the desired result. Further, writing

$$
\left[1-\theta_{n} u\left(1-\sum_{i=1}^{n-1} x_{i}\right)\right]^{-d_{n}}=\sum_{j=0}^{\infty} \frac{\left(d_{n}\right)_{j}\left(\theta_{n} u\right)^{j}}{j !}\left(1-\sum_{i=1}^{n-1} x_{i}\right)^{j}
$$

the joint p.d.f. of $\left(X_{1}, \ldots, X_{n-1}\right)$ and $U$ is rewritten as

$K_{B} u^{\sum_{i=1}^{n} a_{i}-1}(1-u)^{c-\sum_{i=1}^{n} a_{i}-1} \sum_{j=0}^{\infty} \frac{\left(d_{n}\right)_{j}\left(\theta_{n} u\right)^{j}}{j !} \frac{\prod_{i=1}^{n-1} x_{i}^{a_{i}-1}\left(1-\sum_{i=1}^{n-1} x_{i}\right)^{a_{n}+j-1}}{\prod_{i=1}^{n-1}\left(1-\theta_{i} x_{i} u\right)^{d_{i}}}$.

Now, integrating $x_{1}, \ldots, x_{n-1}$ in the above expression by using (2.4), we get the marginal p.d.f. of $U$ as

$$
\begin{aligned}
& K_{B} u^{\sum_{i=1}^{n} a_{i}-1}(1-u)^{c-\sum_{i=1}^{n} a_{i}-1} \sum_{j=0}^{\infty} \frac{\left(d_{n}\right)_{j}\left(\theta_{n} u\right)^{j}}{j !} \frac{\prod_{i=1}^{n-1} \Gamma\left(a_{i}\right) \Gamma\left(a_{n}+j\right)}{\Gamma\left(\sum_{i=1}^{n} a_{i}+j\right)} \\
& \times F_{B}^{(n-1)}\left(a_{1}, \ldots, a_{n-1}, d_{1}, \ldots, d_{n-1} ; \sum_{i=1}^{n} a_{i}+j ; \theta_{1} u, \ldots, \theta_{n-1} u\right), 0<u<1 .
\end{aligned}
$$

Finally, re-writing the infinite series by applying (2.3), we get the desired result.

By definition, the product moments are obtained as

$$
\begin{aligned}
E\left[\prod_{i=1}^{n} U_{i}^{r_{i}}\right] & =\int_{\substack{u_{1}>0, \ldots, u_{n}>0 \\
\sum_{i=1}^{n} u_{i}<1}} \frac{\prod_{i=1}^{n} u_{i}^{a_{i}+r_{i}-1}\left(1-\sum_{i=1}^{n} u_{i}\right)^{c-\sum_{i=1}^{n} a_{i}-1}}{\prod_{i=1}^{n}\left(1-\theta_{i} u_{i}\right)^{d_{i}}} \prod_{i=1}^{n} \mathrm{~d} u_{i} \\
& =\frac{\Gamma(c) \prod_{i=1}^{n} \Gamma\left(a_{i}+r_{i}\right)}{\Gamma(c+r) \prod_{i=1}^{n} \Gamma\left(a_{i}\right)}
\end{aligned}
$$




$$
\times \frac{F_{B}^{(n)}\left(a_{1}+r_{1}, \ldots, a_{n}+r_{n}, d_{1}, \ldots, d_{n} ; c+r ; \theta_{1}, \ldots, \theta_{n}\right)}{F_{B}^{(n)}\left(a_{1}, \ldots, a_{n}, d_{1}, \ldots, d_{n} ; c ; \theta_{1}, \ldots, \theta_{n}\right)}
$$

where $r=\sum_{i=1}^{n} r_{i}, \operatorname{Re}\left(a_{i}+r_{i}\right)>0, i=1, \ldots, n$ and $\operatorname{Re}(c+r)>0$. Further

$$
\begin{aligned}
E\left[\left(1-\sum_{i=1}^{n} U_{i}\right)^{h}=\right. & \int_{\substack{u_{1}>0, \ldots, u_{n}>0 \\
\sum_{i=1}^{n} u_{i}<1}} \frac{\prod_{i=1}^{n} u_{i}^{a_{i}-1}\left(1-\sum_{i=1}^{n} u_{i}\right)^{c+h-\sum_{i=1}^{n} a_{i}-1}}{\prod_{i=1}^{n}\left(1-\theta_{i} u_{i}\right)^{d_{i}}} \prod_{i=1}^{n} \mathrm{~d} u_{i} \\
= & \frac{\Gamma(c) \Gamma\left(c+h-\sum_{i=1}^{n} a_{i}\right)}{\Gamma(c+h) \Gamma\left(c-\sum_{i=1}^{n} a_{i}\right)} \\
& \times \frac{F_{B}^{(n)}\left(a_{1}, \ldots, a_{n}, d_{1}, \ldots, d_{n} ; c+h ; \theta_{1}, \ldots, \theta_{n}\right)}{F_{B}^{(n)}\left(a_{1}, \ldots, a_{n}, d_{1}, \ldots, d_{n} ; c ; \theta_{1}, \ldots, \theta_{n}\right)} .
\end{aligned}
$$

\section{Acknowledgments}

The research work of DKN and AG was supported by the Sistema Universitario de Investigación, Universidad de Antioquia under the project no. IN10182CE.

\section{References}

[1] S. Kotz, N. Balakrishnan and N.L. Johnson, Continuous Multivariate Distributions, Vo. 1, Second Edition, John Wiley \& Sons, New York, USA (2000).

[2] A.K. Gupta and D.K. Nagar, Matrix Variate Distributions, Chapman \& Hall/CRC, Boca Raton, FL, USA (2000).

[3] D. L. Libby and M. R. Novic, Multivariate generalized beta distributions with applications to utility assessment, J. Educ. Statist, 7 (1982), 271-294. URL:http://dx.doi.org/10.2307/1164635

[4] S. Nadarajah, The bivariate $F_{3}$-beta distribution, Commun. Korean Math. Soc., 21 (2006), 363-374.

[5] Daya K. Nagar and Erika Alejandra Rada-Mora, Properties of multivariate beta distributions, Far East J. Theor. Stat., 24 (2008), 73-94. 
[6] T. and Pham-Gia and Q. P. Duong, The generalized beta- and Fdistributions in statistical modelling, Math. Comput. Modelling, 12 (1989), 1613-1625. URL:http://dx.doi.org/10.1016/0895-7177(89)90337-3

[7] A. P. Prudnikov, Yu. A. Brychkov and O. I. Marichev, Integrals and Series. Vol. 3. More special functions. Translated from the Russian by G. G. Gould, Gordon and Breach Science Publishers, New York, USA (1990).

[8] H.M. Srivastava and P.W. Karlsson, Multiple Gaussian Hypergeometric Series, John Wiley \& Sons, New York, USA (1985). 